\title{
How do you Feel About this Goal? Goal-Related Affect, Positive Orientation, and Personal Goal Realization in the Family Domain
}

\author{
Mariola Laguna ${ }^{1}$ Guido Alessandri ${ }^{2}$ - Gian Vittorio Caprara ${ }^{2}$
}

Published online: 12 May 2016

(C) The Author(s) 2016. This article is published with open access at Springerlink.com

\begin{abstract}
Past research on individual-level differences has revealed associations between affect, positive beliefs and goal realization. Goal realization, however, varies not only between individuals (the individual level) but also within individuals according to goal (the goal level). This study analyzed family-related personal goals at both levels, examining how positive affect, negative affect and positive orientation are related to personal goal realization. The participants were 205 adults (mean age $=35.74$ years); they evaluated five personal goals related to family life and completed assessments of positive orientation. Multilevel structural equation modeling demonstrated that both at the individual level and at the goal level positive orientation and positive goal-related affect were positively related to personal goal realization in family domain. Negative goal-related affect was negatively related to personal goal realization at both levels. Positive orientation, both at the individual level and as a cross-level interaction, moderated the relationship between positive goal-related affect and personal goal realization.
\end{abstract}

Keywords Personal goals - Goal realization - Goal-related affect · Positive orientation · Family $\cdot$ Multilevel analysis

\section{Introduction}

Positive affect makes people more likely to engage in actions directed at fulfilling life goals (Carver and Scheier 1999; Plemmons and Weiss 2013), but is this true of all goals for which a person strives? Every day individuals are likely to formulate many different goals

Mariola Laguna

laguna@kul.pl

1 Institute of Psychology, The John Paul II Catholic University of Lublin, Al. Raclawickie 14, 20-950 Lublin, Poland

2 "Sapienza", University of Rome, Rome, Italy 
and many of them are related to family life (Salmela-Aro, Aunola, and Nurmi 2007). These goals are usually accompanied by different affects and the extent to which they are realized will vary. Our knowledge about the relationships between affect, beliefs and goal realization comes from research analyzing these phenomena at the individual-level, i.e. as general individual tendencies. The implicit assumption is that within individuals the process of goal realization is constant across a broad variety of goals, although it varies between individuals. What is missing, however, is an understanding of the mechanisms associated with an individual's pursuit of his or her various goals. We propose in this paper that goal realization varies not only between individuals but also across the various personal goals pursued by each individual. This further level of variability has been neglected in much past research.

With the aim of providing a more nuanced understanding of goal realization in the family domain this study used a multilevel approach (Lüdtke and Trautwein 2007; Nezlek 2012) which enabled us to explore the relationships among affect, personal beliefs and engagement in goal realization at the goal level. More specifically, this approach enabled us not only to investigate the relationships between a tendency to experience positive as well as negative affect towards goals and positive cognitive orientation (Caprara et al. 2009) and goal realization, but also the relationships between realization of a specific personal goal and the affect associated with that goal. This allows us to extend knowledge gained from a between-individual perspective to within-individual phenomena (Cervone 2005). Recent research has shown that the relationships between variables at between- and within-individual levels may be entirely different (e.g., Sitzmann and Yeo 2013).

We begin our introduction by describing how we conceptualize personal goals and explaining why it is important to take into account the multilevel structure of personal goals when analyzing them. Having introduced basic concepts relating to levels of analysis we next discuss potential relationships between affect and goal realization and between positive orientation and goal realization. We then set out the study hypotheses, which relate to the multilevel structure of personal goals.

\subsection{The Nature of Personal Goals}

Goals are cognitive representations of desired end states, and are related to motivational and affective processes (Carver and Scheier 1999; Plemmons and Weiss 2013). People usually pursue multiple goals; from early adulthood these include goals related to work and to family life (Salmela-Aro 2009; Salmela-Aro et al. 2007). Realization of work-related goals had been analyzed in many studies (see Plemmons and Weiss 2013); however family-related goals have received much less attention, although they are as important to people as work-related goals (Salmela-Aro 2009). Longitudinal research indicates that the number of family-related goals an individual formulates increases throughout early adulthood and that these goals have a major influence on actions (Salmela-Aro et al. 2007). Realization of goals related to family is determined both by individuals' and by goals' characteristics (Laguna et al. 2016).

In this paper we focus on personal goals (Little 2006) i.e. goals that are perceived as personally meaningful and important. Personal goals are conceived as intentional, "personally salient" (Little 2006, p. 423) and treated as dynamic motivational entities that direct individuals' behavior. Goals relate directly to what people are trying to do in particular life situations (Little 1999, 2006; McGregor et al. 2006).

According to Little (2006) realizing personal goals requires mental engagement as well as engagement in actions. To move towards a goal an individual necessarily has to 
undertake some activities (Heckhausen and Gollwitzer 1987; Little and Gee 2007). The goal realization process starts when a person formulates a positive outcome expectation (positive subjective evaluation of goal attainability) and creates a clear action plan (implementation intentions, Gollwitzer 1999). Progress through the stages of the goal attainment process should be monitored (Heckhausen and Gollwitzer 1987) and the person must remain engaged in action until the desired outcome has been reached (Carver and Scheier 1999; Little and Gee 2007). In other words progress towards a goal depends jointly on outcome expectations, clear plans, monitoring of stages of project realization and engagement in action. We follow Laguna et al. (2016) in treating these four components as key to personal goal realization. Progress towards personal goal realization is important to individuals' subjective wellbeing (Steca et al. 2015).

\subsection{Multilevel Structure of Personal Goals}

Personal goals, as defined above, are usually assessed in a two-step procedure (Little and Gee 2007). In the first step participants write down the goals that are meaningful to them. In the second step they rate some characteristics of these goals. This procedure is such that it combines idiographic (i.e., capturing what is unique to an individual) and nomothetic (i.e., capturing what is common to a number of people) approaches to personality assessment (Lamiell 1981). Like idiographic approaches it takes into account the individuality of personal goals, and like nomothetic approaches it allows between-individual comparisons of goals in terms of common appraisal dimensions (Lüdtke and Trautwein 2007).

As one person may be engaged in pursuit of several different goals at the same time (e.g., collecting one's child from school, buying vegetables for dinner, being friendly to people) personal goals have a natural multilevel structure (Lüdtke and Trautwein 2007). The first level of this structure (i.e. Level 1) is the goal level; an individual's various personal goals are described at this level. This first level is nested within a second, more general level (i.e. Level 2) at which goals differ between individuals, this level is called the 'individual' level. This nested structure is necessary to capture the fact that individuals have several different goals (described at the within-individual level, Level 1) and that personal goals also differ between individuals (captured at the between-individual level, Level 2). Unfortunately, most research on personal goals to date has focused only on the individual level (Level 2), and has relied on aggregate measures of an individual's different goals (cf. Little 1999; Sheldon and Elliot 2000; Wiese and Freund 2005), which distorts the within-individual differences between goals. A multilevel framework (Nezlek 2007, 2012) allows us to consider within- and between-individual variance in goals separately.

It is important to note that multilevel analysis of personal goals offers an opportunity to determine whether findings obtained in a between-individual context are replicable when within-individual differences in goals are also taken into account. An issue in much of the past research is that many of the conclusions about goal-level phenomena have been drawn from data analyzed at the individual level (Boudreaux and Ozer 2013). Taking the multilevel approach described above allows us to overcome these shortcomings and has the potential to reveal whether the mechanisms of goal realization are generalizable not only across individuals but also across different goals within an individual.

Other advantages associated with this framework are (1) it allows relationships between goal-related affect and goal realization to be studied within individuals (at the goal level) whilst also taking into account differences between individuals (i.e., the individual level) and (2) it makes it possible to consider interactions between the goal and the individual 
levels (Nezlek 2012). More generally, the multilevel approach makes it possible to analyze so-called personality architecture - the characteristics of within-individual personality systems and processes (Cervone 2005).

As stated above, there is still little published research taking into account the multilevel perspective (Nurmi et al. 2009; Salmela-Aro et al. 2011) although it has been strongly recommended (Lüdtke and Trautwein 2007). Research on employees showing that a decrease in burnout symptoms is associated with a decrease in the negative affect and an increase in the positive affect related to work and interpersonal goals (Salmela-Aro et al. 2011) illustrated the potential dividends of multilevel research. Other research showed that personal goal accomplishment is negatively associated with both depressive symptoms and stress (Nurmi et al. 2009). These successful applications of a multilevel framework in research on personal goals indicate that it may improve understanding of the relationships between affect, beliefs and family goal realization.

\subsection{Affect and Goal Realization}

Many studies have confirmed that affect (mood and emotions) is related to progress towards a goal and success in realizing goals (Plemmons and Weiss 2013; Wiese and Freund 2005). Affect may, however, be a source of goal engagement and not only a byproduct of movement toward goals. It has been documented that affect is integrated with goal realization in several ways: it influences goal choice, focuses the individual's attention on the goal, helps determine the expectancy of goal attainment and determines persistence (for a review see Plemmons and Weiss 2013). One of the functions of affect is to direct and energize goal-directed behavior and it is a better predictor of behavior than cognitive evaluations (Bagozzi et al. 2000). Several studies have confirmed that affective processes engender volitional processes related to formation of behavioral intentions, decisions about investment of effort in goal pursuit and planning and monitoring of goal realization (see for a review Bagozzi et al. 2000). We agree with Seo et al. (2010, p. 962) summary of this research: "[affect] is an important motivational property that systematically predicts behavioral choice, intensity, and persistence".

Positive and negative affect play important but different role in self-regulatory processes related to goal realization (Baumeister et al. 2007; Carver and Scheier 1999); however our understanding of the role of affect in family-related goal realization is limited (see for example Judge et al. 2006). There is evidence that positive affect broadens an individuals' thought-action repertoires (Fredrickson 2001) thus prompting them to engage in activities that may promote goal realization. The evidence concerning negative affect is less consistent. Some kinds of negative affect signal insufficient progress and may cause goal disengagement (e.g., depression) whereas others (e.g., anger) can foster goal realization (Carver and Scheier 1999). The widely used circumplex model of affect defines affective states as cognitive interpretations of neural sensations that are the product of two fundamental neurophysiological systems: valence (a pleasure-displeasure continuum) and arousal (Russell 1980). This model is well supported by findings from neuroscience, neuroimaging, behavioral research and developmental studies (Posner et al. 2005). A specific affective state is thus a linear combination of these two dimensions, pleasantness and activation level. In our study we considered affective states from all four parts of the affective circle of the circumplex model (see figure in Russell 1980, p. 1164). As goal realization is thought to be related to high rather than low arousal (Carver and Scheier 1999) we investigated two positive and two negative affective states associated with high arousal (curiosity and enthusiasm and anxiety and anger respectively) and one low-arousal 
affective indicator of each pole of the pleasure-displeasure continuum (contentment and depression).

Personal goals are closely related to affect (Little 2006) but the levels of positive and negative affect an individual associates with his or her various goals can vary across goals. Researchers have demonstrated the existence of affective states associated with a specific goal, referred to as goal-related affect (Laguna et al. 2016). The multilevel framework allows us to assess the affect accompanying each goal (at the goal level, Level 1), and then to obtain an aggregate (across many different goals) measure of positive or negative affect at the individual level (Level 2).

Considered from an individual differences perspective previous studies show that positive affect increases goal engagement (Plemmons and Weiss 2013). The few studies that have investigated goal-related affect show that a decrease in negative affect or an increase in positive affect has a beneficial effect on employees' functioning at work (Pomaki et al. 2004; Salmela-Aro et al. 2011) and on entrepreneurs (Laguna et al. 2016). The aim of this study was to extend understanding of the relationship between goal-related affect and family goal realization by approaching it from a multilevel perspective. Based on the research discussed above we formulated the following hypothesis:

Hypothesis 1 Positive goal-related affect is positively related to personal goal realization in the family domain both at the individual level (Hypothesis 1a) and at the goal level (Hypothesis 1b).

In view of the inconsistent evidence on the relationships between negative affect and goal realization we also asked:

Research Question 1 What is the relationship between negative goal-related affect and personal goal realization in the family domain at the individual level (Research Question la) and at the goal level (Research Question 1b)?

\subsection{Positive Orientation and Goal Realization}

Successful goal realization is likely to be related not only to affect but also to the beliefs a person holds about his or her relationship with the world (Carver and Scheier 1999). Moderate positive expectations for oneself and of events and the world more generally tend to inspire greater confidence and greater persistence in pursuit of goals. On the other hand negative selfbeliefs cause effort reduction or goal disengagement (Carver and Scheier 1999). As many studies have demonstrated, being positively oriented toward oneself (self-esteem), one's life (life satisfaction) and towards the future (dispositional optimism) is generally associated with wellbeing and success across a variety of domains of functioning (Baumeister et al. 2003; Lyubomirsky et al. 2005; Scheier et al. 1994). It is likely that these same general beliefs are also related to personal goal realization in the family domain as there is some evidence that self-esteem is related to commitment to personal goals (Zięba 2012). Furthermore, optimism is associated with goal commitment and with progress towards goal realization via the higher goal expectancy (Monzani et al. 2015). Optimism has also been shown to be related to flexible goal adjustment, and through this to wellbeing (Hanssen et al. 2016).

It has been shown that all three beliefs mentioned above - self-esteem, life satisfaction, and dispositional optimism - reflect an individual's general tendency to approach reality in a positive way (Caprara et al. 2009, 2010, 2016; Oleś et al. 2013). They are therefore treated as components of positive orientation (POS), called also positivity. POS is a higher order construct that captures the core of self-esteem, life satisfaction and optimism and its 
validity has been confirmed empirically (Caprara et al. 2009, 2010; Oleś et al. 2013). This cognitive triad (which can be contrasted with the depressive triad; Beck 1967) leads people to hold a positive view of themselves, life and the future, making them willing to persist in the face of obstacles and failures (Caprara et al. 2009). Genetic studies (Caprara et al. 2009) converge with longitudinal and cross-sectional findings (Caprara et al. 2010, 2016) in showing that POS is a basic predisposition that accounts for a considerable proportion of the variance in individuals' adjustment and achievement (Caprara et al. 2009). Empirical studies have demonstrated that POS is related to optimal functioning in several life domains (Caprara et al. 2010) and to objective work achievement, when controlling for basic individual dispositions and positive affectivity (Alessandri et al. 2012). POS is also correlated with a generally positive attitude towards time and an active focus on the present (Sobol-Kwapinska and Jankowski 2015) that may lead to greater openness to alternative solutions when the path to realization of a specific goal is blocked. Based on previous research we hypothesized that:

Hypothesis 2 POS is positively related to personal goal realization in the family domain at the individual level.

We further hypothesized that affect and generalized positive beliefs interact in the goal realization. This hypothesis was based on (1) research indicating that positive general beliefs can intensify the experience of positive affect and, conversely, reduce the pernicious consequences of negative affect (Bandura 2001; Carver and Scheier 1999), (2) previous studies indicating that positive self-evaluations are significant moderators of the relationship between affect and pursuit of goals (Ilies et al. 2007; Laguna et al. 2016).

Whereas earlier research analyzed the relations between beliefs and affect at the individual level (e.g., Salanova et al. 2011), the multilevel framework also allowed us to examine this interaction at the goal level (i.e., a cross-level effect). To illustrate this interaction between general beliefs and goal-related affect, we refer to research findings which show that POS is accompanied by a dispositional tendency to experience positive affect (Alessandri et al. 2012). Although people high in POS experience negative affect in relation to some goals their POS may serve as a buffer against disengagement from the goals concerned (Laguna et al. 2016). We therefore hypothesized that POS is a moderator of the relationship between goal-related affect and goal realization:

Hypothesis 3.1 The positive relationship between positive goal-related affect and personal goal realization in the family domain is stronger when a person has higher POS, and (Hypothesis 3.2) the negative relationship between negative goal-related affect and personal goal realization in the family domain is weaker when a person has higher POS. Both effects are hypothesized to apply to the individual level (Hypothesis 3.1a and Hypothesis $3.2 a$ ) and as cross-level interactions (Hypothesis 3.1b and Hypothesis 3.2b).

\section{Method}

\subsection{Procedure}

Data were gathered during individual meetings with people invited to take part in the study by e-mail and personal contact. Snowball sampling was used - the participants were asked to provide contact details for other potential respondents. We used marital status as a selection criterion: participants had to be (1) either married or in a stable relationship with 
a partner, (2) of at least two years' standing. Following recommendations made in previous studies (McGregor et al. 2006, study 1) the participants filled out the paper and pencil version of Personal Project Analysis (PPA) first and then completed the other measures. Participants did not receive any reward for taking part in the study. Most of them found the procedure an interesting experience which gave them insight into their personal goals. Participants were assured that their data would remain anonymous and confidential.

\subsection{Participants}

We collected data from 211 adults, data from six individuals were excluded due to missing values, leaving a final sample of 205 adults aged $19-68$ years $(M=35.74, S D=10.37)$ of whom $101(47.9 \%)$ were women. All participants were married $(n=147,72 \%)$ or in a stable relationship with a partner $(n=58,28 \%)$. Most of them had children, 51 participants $(24.9 \%)$ had one child, $55(26.8 \%)$ two, $20(9.8 \%)$ three or more and $79(38.5 \%)$ did not have children. The distribution of educational attainment was as follows: 123 $(60 \%)$ had completed higher education; 68 (33.2\%) had completed high school and 14 $(6.8 \%)$ had completed vocational secondary school. Most participants were full-time employees $(127,62 \%), 32(16 \%)$ were employed part-time, 34 (17\%) were self-employed and $12(6 \%)$ had other forms of work contract (e.g., apprenticeship).

\subsection{Measures}

To assess personal goal realization and goal-related affect we used a modified version of the PPA method (Little and Gee 2007). The PPA method is very flexible, a subset of the modules can be selected and respondents can be asked to rate only the subset of dimensions appropriate to a specific study (e.g., Pomaki et al. 2004; Salmela-Aro et al. 2011). In this study we used two modules (modules 1 and 2) and 10 PPA dimensions relevant to goal realization and positive and negative affect. The selection of dimensions was based on the conceptualizations of goal realization and affect set out in the 'Introduction' section. The respondents first listed five current personal goals related to family (e.g., "to have more time for my family", "to renovate the house"). Then they rated each goal on four dimensions related to goal realization, using eleven-point scales: outcome expectations ( 0 - total failure, 10 - entirely successful), plan clarity ( 0 - have no plan of project realization, 10 - have a clear and concrete plan), stage of goal realization ( 0 - the idea for the project has just come to me, 10 - the project is coming to a close or has actually been completed or terminated), engagement ( 0 - not engaged, 10 - fully engaged $)$. Goal-related affect with respect to three positive affective dimensions (enthusiastic, content, curious) and three negative ones (depressed, anxious, angry) was also rated on an eleven-point scale (from 0 - do not feel it at all, to 10 - very strongly). Data on the reliability of these instruments is presented below in the 'Validity of Measures' section.

We used three scales to assess the three components of the second order factor POS. Self-esteem was assessed using Rosenberg's (1965) ten-item Self-Esteem Scale, which requires respondents to rate items (e.g., "On the whole, I am satisfied with myself") using a four-point scale (1 - strongly disagree, 4 - strongly agree). Higher scores indicate higher self-esteem. Cronbach's $\alpha$ for the scale was .79. Optimism was measured using the ten-item Life Orientation Test (LOT-R; Scheier et al. 1994). Six items of the scale (the other four items are fillers) measure expectations about the future (e.g., "I am always optimistic about my future"); participants indicate the extent to which each item reflects their orientation using a five-point scale (1 - strongly disagree, 5 - strongly agree $)$. Higher scores indicate 
greater optimism. Cronbach's $\alpha$ for this measure was .88. Life satisfaction was assessed using the five-item Satisfaction With Life Scale (SWLS; Diener et al. 1985); respondents indicate the extent to which items (e.g., "I am satisfied with my life") apply to them using a seven-point scale $(1$ - strongly disagree, 7 - strongly agree $)$. Higher scores indicate a higher level of life satisfaction. Cronbach's $\alpha$ for the scale was .74.

\subsection{Data Analysis}

In this study we obtained multilevel data on goals, with 205 observations at the individual level and 1025 observations at the goal level. To determine the extent of between-unit variance in all variables we computed the intraclass correlation coefficient (ICC) and the Design Effect Index (DEF) using procedures devised by Muthen and Satorra (1995). ICC is calculated as the ratio of the maximum likelihood estimates of the latent within- and between-level variance components, assuming random level effects, and ranges in value from 0 to 1 . DEF is a function of average cluster size and intraclass correlation and is computed as DEF $=1+\delta(n-1)$, where $\delta$ is the observed value for ICC, and $n$ is the average cluster size. A DEF greater than 2 indicates that the clustering of data needs to be taken into account. In this study the values of these indices (Table 1) regularly exceeded the recognized thresholds for multilevel analysis (see also 'Validity of Measures' below). Thus, we used multilevel structural equation modeling (Ludtke et al. 2008) to test the hypothesized main and interaction effects at goal and individual levels simultaneously and to include (and then test the appropriateness of) random intercepts, and cross-level interaction terms as random slopes.

All models were specified with Mplus 5.1 using the multilevel latent covariate approach (MLC, Ludtke et al. 2008). All Multilevel Confirmatory Factor Analysis (ML-CFA) models were run on individuals' responses to PPA dimensions. Confirmatory Factor Analysis (CFA) was carried out on individuals' responses to questionnaire items. Model fit was assessed using the $\chi^{2}$ likelihood ratio statistic, Comparative Fit Index (CFI), and the Root Mean Square Error of Approximation (RMSEA). The $\chi^{2}$ statistic is sensitive to sample size and large samples easily produce a statistically significant result (Kline 2012). We accepted CFI values greater than .90 and RMSEA values lower than .08. Non-nested models were compared using the Akaike Information Criteria (AIC), which is appropriate for comparing models with cross-level interaction (see 'Modeling Strategies' section). Lower values of AIC indicate a better model fit (Kline 2012).

\subsection{Validity of Measures}

\subsubsection{Personal Goal Realization}

The ICC and DEF for the four indicators were as follows, outcome expectation: $\mathrm{ICC}=.40, \mathrm{DEF}=2.60$; plan clarity: $\mathrm{ICC}=.51, \mathrm{DEF}=3.04$; stage of goal realization: $\mathrm{ICC}=.35, \mathrm{DEF}=2.40$; engagement: $\mathrm{ICC}=.42, \mathrm{DEF}=3.10$. The moderately high values of ICC in combination with the high values for the DEF indicated that the hierarchical nature of the data would need to be taken into account. We therefore used MLCFA to confirm the hypothesized one-factor structure for our measure of goal realization. The results revealed that the one-factor model was a good fit to the data: $\chi_{(4)}^{2}=31.41$, $p<.01, \mathrm{CFI}=.985, \mathrm{RMSEA}=.053$. At the goal level the completely standardized factor loadings were .76 (outcome expectation), .79 (plan clarity), .59 (stage of goal 
Table 1 Correlations between variables at the goal (below the main diagonal) and at the individual level (above the main diagonal)

\begin{tabular}{lccccc}
\hline Variable & ICC & DEF & \multicolumn{2}{l}{ Correlations } & \\
\cline { 5 - 6 } & & & 1 & 2 & 3 \\
\hline 1. Goal realization & .45 & 3.25 & - & $.41^{* * * *}$ & $-.20^{* * * *}$ \\
2. Positive goal-related affect & .70 & 4.50 & $.39^{* * *}$ & - & $-.17^{* * * *}$ \\
3. Negative goal-related affect & .58 & 3.90 & $-.23^{* * *}$ & -.05 & - \\
4. Positive orientation & - & - & .11 & .08 & $-.14^{*}$ \\
\hline
\end{tabular}

ICC intraclass correlation coefficient; DEF Design Effect Index; we used factor scores (mean = zero and standard deviation $=1$ )

$* p<.05 ; * * * p<.001$

realization) and .76 (engagement); at the individual level they were .85 (outcome expectation), .96 (plan clarity), .62 (stage of goal realization) and .94 (engagement). The factor loadings were significant at both levels $(p<.05)$. Score reliability was assessed using the omega coefficient (see Kline 2012) which had a value of .51 at the goal level and .71 at the individual level.

\subsubsection{Goal-Related Affect}

The ICC and DEF for the three indicators of positive affect were as follows, enthusiastic: $\mathrm{ICC}=.75, \quad \mathrm{DEF}=4.00 ; \quad$ content: $\quad \mathrm{ICC}=.69, \quad \mathrm{DEF}=3.76 ; \quad$ curious: $\quad \mathrm{ICC}=.70$, $\mathrm{DEF}=3.80$. The ICC and DEF for the three indicators of negative affect were as follows, depressed: $\mathrm{ICC}=.61, \mathrm{DEF}=3.44$; anxious: $\mathrm{ICC}=.50, \mathrm{DEF}=3.00$; angry: $\mathrm{ICC}=.63$, $\mathrm{DEF}=3.52$. Again, ML-CFA seemed appropriate. Results from the ML-CFA revealed that a model with two correlated latent factors representing positive and negative affect was a good fit: $\chi_{(18)}^{2}=125.05, p<.01, \mathrm{CFI}=.94, \mathrm{RMSEA}=.049$. At the goal level the completely standardized factor loadings for positive affect were .82 (enthusiastic), .75 (content) and .68 (curious); the corresponding values for negative affect were .80 (depressed), .79 (angry) and .75 (anxious). At the between-individual level the loadings for positive affect were: .99 (enthusiastic), .76 (content) and .67(curious) and the loadings for negative affect were: .96 (depressed), .88 (angry) and .77 (anxious). At both levels the factor loadings were significant $(p<.05)$. The correlation between the latent factors representing positive and negative affect was significant only at the goal level (-.22). At the goal level score reliability was .55 for positive affect and .61 for negative affect; at the individual level it was .79 for positive affect and .76 for negative affect.

\subsubsection{POS}

Following Alessandri et al. (2012), we tested the fit of a CFA model with self-esteem, life satisfaction and optimism as first-order factors loading on a higher-order factor POS. As self-esteem, life satisfaction and optimism were all level 2 variables this analysis did not require ML-CFA. We also included three specific factors (uncorrelated with all other factors) accounting for items wording effects associated with the five positive and the five negative items from the Rosenberg scale, and the three positive items from the LOT-R scale. This model fitted the data very well: $\chi_{(170)}^{2}=655.81, p=.00, \mathrm{CFI}=.962$, 
RMSEA $=.041$. The loadings of self-esteem, life satisfaction and optimism on the latent factor were $.91, .75$, and .89 respectively. Mean loadings for the items associated with the first-order factors were moderately high: $.51(S D=.15)$ for self-esteem; $.42(S D=.11)$ for life satisfaction and $.55(S D=.02)$ for optimism.

\subsection{Modeling Strategies}

To test the hypotheses we sequentially specified three two-level models of personal goal realization including goal-level (Level 1) and individual-level (Level 2) variables (see Table 2 for a further explanation of the variables entered in each model). First we specified a model in which positive and negative goal-related affect were entered as predictors at both the individual level and the goal level and POS was entered as a predictor at the individual level (Model 1). Next (in Model 2) we tested the hypothesized moderation by POS of the relationships between affect and goal realization at the individual level. To do so we included, at Level 2, two additional terms representing the interactions between POS and positive and negative affect alongside the terms representing the main effects of positive and negative goal-related affect and POS. Finally, to investigate this moderation effect at the goal level we specified a third model (Model 3). In this model, we added two cross-level interactions between POS and goal-related affect at the goal level (I-POS*GPositive affect and I-POS*G-Negative affect) to all the variables included in Model 2 (see Table 2). In all these analyses we used factor scores derived from previous ML-CFA and CFA models as observed variables. These scores were expressed as standardized $z$ scores.

\section{Results}

The results of the multilevel analyses are presented in Table 2. In Model 1 personal goal realization was positively predicted by positive goal-related affect and negatively predicted by negative goal-related affect at both goal level and individual level. In addition POS

Table 2 Unstandardized estimates (B) and standard errors (SE) for predictors of personal goal realization

The letter 'I' denotes individual level variables; the letter ' $G$ ' denotes goal level variables

POS positive orientation; PosAff goal-related positive affect;

NegAff goal-related negative affect; AIC Akaike Information Criteria

$* p<.05$

\begin{tabular}{|c|c|c|c|c|c|c|}
\hline \multirow[t]{2}{*}{ Predictors } & \multicolumn{2}{|c|}{ Model 1} & \multicolumn{2}{|c|}{ Model 2} & \multicolumn{2}{|c|}{ Model 3} \\
\hline & $B$ & $S E$ & $B$ & $S E$ & $B$ & $S E$ \\
\hline \multicolumn{7}{|l|}{ Individual level } \\
\hline I-Positive Affect & $.33^{*}$ & .06 & $.29 *$ & .03 & $.15^{*}$ & .08 \\
\hline I-Negative Affect & $-.09 *$ & .03 & $-.15^{*}$ & .05 & $-.08 *$ & .04 \\
\hline I-POS & $.16^{*}$ & .08 & $.16^{*}$ & .07 & $.11 *$ & .04 \\
\hline I-POS*I-PosAff & - & - & $.07 *$ & .03 & $.07 *$ & .03 \\
\hline I-POS*I-NegAff & - & - & .01 & .03 & .01 & .01 \\
\hline \multicolumn{7}{|l|}{ Goal level } \\
\hline G-Positive Affect & $.31 *$ & .08 & $.32 *$ & .08 & $.30 *$ & .09 \\
\hline G-Negative Affect & $-.15^{*}$ & .06 & $-.13 *$ & .06 & $-.12 *$ & .06 \\
\hline \multicolumn{7}{|c|}{ Cross level interaction } \\
\hline I-POS*G-PosAff & - & - & - & - & $.04 *$ & .01 \\
\hline I-POS*G-NegAff & - & - & - & - & .01 & .01 \\
\hline \multicolumn{7}{|l|}{ Model fit } \\
\hline AIC & 23657.77 & \multicolumn{2}{|c|}{23652.89} & \multicolumn{2}{|c|}{23650.01} & \\
\hline
\end{tabular}


positively predicted personal goal realization at the individual level. In Model 2, at the individual level, there were main effects of positive goal-related affect, negative goalrelated affect and POS, and the interaction between POS and individual-level goal-related positive affect also predicted goal realization. More specifically, the relationship between goal realization and positive goal-related affect was stronger in more positively oriented individuals. The individual-level interaction between negative goal-related affect and POS did not predict goal realization. Model 3 indicated that at the goal level the relationship between goal realization and positive goal-related affect was moderated by $\operatorname{POS}^{1}$. Accordingly, for more positively oriented individuals, the relationship between positive goal-related affect and goal realization was stronger also at the goal level. POS did not moderate the relationship between negative goal-related affect and goal realization at the goal level. Model comparisons (see AIC in Table 2) showed that Model 3 was the best fitting model. Hypotheses 1, 2 and 3.1 were confirmed at the individual level (Hypothesis 1a, Hypothesis 3.1a) and at the goal level (Hypothesis 1b, Hypothesis 3.1b). The results also indicated that negative goal-related affect was negatively related to personal goal realization both at the individual level (Research Question la) and at the goal level (Research Question $1 b$ ). There was no interaction between negative goal-related affect and POS at either the individual level (Hypothesis 3.2a) or the goal level (Hypothesis 3.2b).

In a supplementary analysis, we checked the role of specific positive and negative goalrelated affects. We did not test these specific effects using MLC approach due to multicollinearity among these variables. We calculated correlations between six specific goalrelated affects and goal realization. They generally mimicked the results of the analysis of the general effects of positive and negative goal-related affect. With regard to specific positive affects, enthusiastic showed the highest positive correlation with goal realization at both levels (.41 at the goal level and .39 at the individual level); the corresponding figures were slightly lower for content (.39 at the goal level and .32 at the individual level), and the lowest for curious (.22 at the goal level and .28 at the individual level). All of them were statistically significant $(p<.001)$. As regards specific types of negative affect, the highest negative correlation with goal realization was found for angry $(-.21$ at the goal level and -.22 at the individual level); depressed (-.20 at the goal level and -.19 at the individual level), and anxious (-.11 at the goal level and -.18 at the individual level) showed lower correlations, all being statistically significant (at least $p<.01$ ).

\section{Discussion}

Personal goals can be treated as a multilevel construct (Lüdtke and Trautwein 2007) and this study investigated relationships between goal-related affect and personal goal realization at the goal level and at the individual level. We also investigated whether the relationship between goal-related affect and personal goal realization was moderated by positive beliefs, namely POS (Caprara et al. 2009, 2010, 2016). The results confirmed that at the goal level and at the individual level positive and negative goal-related affect have opposite effects on personal goal realization in the family domain. Individuals' efforts to achieve particular goals are positively related to positive goal-related affect and negatively

\footnotetext{
1 The variance associated with the cross-level interaction involving affect at the goal level and POS at the individual level was statistically significant for positive goal-related affect (for I-POS*G-Positive Affect it was $.01^{*}, S E<.001$ ), and for negative goal-related affect it was not statistically significant (for I-POS*GNegative Affect it was .01, $S E=.04)$.
} 
related to negative affect. In other words positive and negative affect respectively enhance and diminish general tendency for activity engagement, as longitudinal studies have demonstrated (e.g., Salanova et al. 2011); however we also established that at the goal level the relationships follow the same pattern. The higher the enthusiasm for, curiosity about and contentedness with a specific goal, the greater one's efforts to realize this goal, whereas the greater the depression, anxiety and anger one feels about a personal goal the less effort one makes to achieve it (Wrosch et al. 2003).

The multilevel approach offered an opportunity to reveal further the mechanisms of goal realization. Like another multilevel analysis of affective states and traits (Nezlek 2007), this study provides evidence about the relationships between event-specific and generalized affect and goal realization. Moreover, variability of goal realization as well as of goal-related affect at the individual and at the goal level justifies further the usefulness of multilevel framework in personal goals analyses. This is consistent with previous research (Laguna et al. 2016; Nurmi et al. 2009); taken together these studies show that although goal appraisals differ between individuals, they also vary greatly across the various goals pursued by a given individual. It is worth noting, however, that the correlation between positive affect experienced towards different family-related goals within individuals in this study is relatively high, and much higher than in the study of Nurmi et al. (2009). This shows that positive affect associated with goals related to family life is more consistent than positive affect related to goals from different life domains.

Additional analyses showed that the valence of affective states (Posner et al. 2005; Russell 1980) is systematically related to goal realization regardless of activation level. These relationships followed similar patterns at the individual level and the goal level. This suggests that the pleasure-displeasure continuum of the circumplex model of affect (Russell 1980) is more important to goal realization than the arousal continuum. Our research also demonstrates that the relationship between positive goal-related affect and family goal realization can be enhanced by high POS, at both the individual level and the goal level. In people higher in POS experience of goal-related positive affect is closely related to efforts to realize goals. As POS increases tendency to focus on the present moment (Sobol-Kwapinska and Jankowski 2015) it may help to translate momentary, positive emotional experiences into goal-directed actions and efforts to deal with obstacles (Fredrickson 2001).

It is interesting that POS was positively associated with personal goal realization, but did not attenuate the relationship between negative affect and goal realization. This confirms the findings of research on entrepreneurs (Laguna et al. 2016). It appears that even high POS people put less effort into realizing a goal which elicits negative affect. A tendency to approach life from a positive perspective does not serve as a buffer against the detrimental effect of negative affect on goal realization (Carver and Scheier 1999). In summary, POS seems to act as a general adaptive tendency (Caprara et al. 2009, 2010) which does not distort the self-regulatory mechanism through which negative affect protects individuals against expending effort pursuing unattainable goals (Wrosch et al. 2003). These findings have theoretical relevance as they clarify the role of POS in self-regulatory processes.

This study adds to existing research on the role of positive goal-related affect (Laguna et al. 2016; Salmela-Aro et al. 2011), providing evidence concerning goals related to family life, which were listed and evaluated by the participants in this study. It is possible, however, that in other contexts the relationships between goal-related affect and goal realization may be different, as shown in research on entrepreneurs and their work-related goals (Laguna et al. 2016). 


\subsection{Limitations and Avenues for Future Research}

Although it provides new evidence on personal goal realization our study has some limitations. The cross-sectional design means it is not possible to establish the causal relationships between the variables of interest. Previous studies on work engagement (Salanova et al. 2011) show that reciprocal relationships between personal beliefs, affect and engagement are possible. Longitudinal research to investigate causality would be valuable as would including behavioral indicators of goal engagement.

We did not take into account the hierarchical nature of goals in this study. People pursue multiple goals and these goals are hierarchically organized (Little and Gee 2007). Often the achievement of low-level, concrete, short-term goals (e.g., collecting one's child from school) contributes to accomplishment of higher level, more abstract, longer term goals (e.g., to be a good parent). Even within a multilevel framework it is not easy to investigate the hierarchical structure of goals and this remains a task for future studies.

We also did not distinguish between extrinsically and intrinsically motivated goals. Whilst personal goals are assumed to be personally significant (Little 2006) the extent to which they are a matter of individual choice may vary. Autonomous motivation is substantially related to progress in goal realization whereas controlled motivation is not (Koestner et al. 2008). Moreover, the extent to which people adopt autonomous goals depends on a need satisfaction in a given domain (Milyavskaya et al. 2014). It is possible that the relationships between goal realization and goal-related affect and beliefs vary depending on whether the goal is pursued out of personal interest or was imposed by others. This is an interesting avenue for future studies.

This study concentrated on POS as a set of positive beliefs (Caprara et al. 2009, 2010, 2016) which plays an important role in goal realization. There is however another widely studied set of positive beliefs, namely self-efficacy beliefs, which may be related to goal realization. Social-cognitive theory defines self-efficacy as one's conviction of one's capability of executing the action required to obtain a desired outcome (Bandura 2001). Self-efficacy is correlated with POS (Oleś et al. 2013) and reciprocally related to positive affect, activity (Salanova et al. 2011) and performance (Sitzmann and Yeo 2013), so it may play a similar role in relationships between goal-related affect and goal realization to POS. This suggestion awaits examination.

\subsection{Conclusions and Implications for Practice}

Despite the above limitations the simultaneous analysis of within- and between-individual phenomena provides new insight into the complexity of individual actions (Cervone 2005). Our findings are potentially useful to counselors, coaches or other professionals working with individuals to help them achieve specific goals. There is evidence that psychological interventions can stimulate individuals to attain personal goals (Sheldon et al. 2002). Interventions designed to strengthen positive beliefs and improve emotion regulation strategies (Gross 1998) may help individuals to engage in actions which will lead to the attainment of personally valuable goals. However it may be helpful to develop goalspecific emotion regulation strategies as well as more general mood regulation strategies. The PPA method can be successfully incorporated into counseling and coaching sessions (Little and Gee 2007) to increase individuals' awareness of personal goal priorities and extend their awareness of specific affective states associated with specific goals and with each stage of the process of their realization. Participants in this study found the PPA 
method interesting and helpful, and commented that it gave them insight into their goals. The personal goals theory (Little 1999, 2006; McGregor et al. 2006) offers a suitable theoretical framework for the development of practical interventions of this kind.

Open Access This article is distributed under the terms of the Creative Commons Attribution 4.0 International License (http://creativecommons.org/licenses/by/4.0/), which permits unrestricted use, distribution, and reproduction in any medium, provided you give appropriate credit to the original author(s) and the source, provide a link to the Creative Commons license, and indicate if changes were made.

\section{References}

Alessandri, G., Vecchione, M., Tisak, J., DeIana, G., Caria, S., \& Caprara, G. V. (2012). The contribution of positivity to predict job performance and organizational citizenship behaviors. Applied Psychology: An International Review, 62, 669-698. doi:10.1111/j.1464-0597.2012.00511.

Bagozzi, R. P., Baumgartner, H., Pieters, R., \& Zeelenberg, M. (2000). The role of emotions in goal-directed behavior. In S. Ratneshwar, D. G. Mick, \& C. Hoffman (Eds.), The why of consumption: contemporary perspectives on consumer motives, goals and desires (pp. 36-58). New York: Routledge.

Bandura, A. (2001). Social cognitive theory: An agentic perspective. Annual Review of Psychology, 52, 1-26. doi:10.1146/annurev.psych.52.1.1.

Baumeister, R. F., Campbell, J. D., Krueger, J. I., \& Vohs, K. D. (2003). Does high self-esteem cause better performance, interpersonal success, happiness, or healthier lifestyle? Psychological Science in the Public Interest, 4, 1-44. doi:10.1111/1529-1006.01431.

Baumeister, R. F., Zell, A. L., \& Tice, D. M. (2007). How emotions facilitate and impair self-regulation. In J. J. Gross (Ed.), Handbook of emotion regulation (pp. 408-426). New York: Guilford Press.

Beck, A. T. (1967). Depression: Clinical, experimental and theoretical aspects. New York: Hoeber.

Boudreaux, M. J., \& Ozer, D. J. (2013). Goal conflict, goal striving, and psychological well-being. Motivation and Emotion, 37, 433-443. doi:10.1007/s11031-012-9333-2.

Caprara, G. V., Eisenberg, N., \& Alessandri, G. (2016). Positivity: The dispositional basis of happiness. Journal of Happiness Studies. doi:10.1007/s10902-016-9728-y.

Caprara, G. V., Fagnani, C., Alessandri, G., Steca, P., Gigantesco, A., Cavalli Sforza, L. L., et al. (2009). Human optimal functioning: The genetics of positive orientation towards self, life, and the future. Behaviour Genetics, 39, 277-284. doi:10.1007/s10519-009-9267-y.

Caprara, G. V., Steca, P., Alessandri, G., Abela, J. R. Z., \& McWhinnie, C. M. (2010). Positive orientation: Explorations on what is common to life satisfaction, self-esteem, and optimism. Epidemiologia $e$ Psichiatria Sociale, 19, 63-71. doi:10.1017/S1121189X00001615.

Carver, C. S., \& Scheier, M. F. (1999). Stress, coping, and self-regulatory processes. In L. A. Pervin \& O. P. John (Eds.), Handbook of personality: theory and research (pp. 553-575). New York: Guilford Press.

Cervone, D. (2005). Personality architecture: Within-person structures and processes. Annual Review of Psychology, 56, 423-452. doi:10.1146/annurev.psych.56.091103.070133.

Diener, E., Emmons, R. A., Larsen, R. J., \& Griffin, S. (1985). The satisfaction with life scale. Journal of Personality Assessment, 49, 71-75. doi:10.1207/s15327752jpa4901_13.

Fredrickson, B. L. (2001). The role of positive emotions in positive psychology: The broaden-and-build theory of positive emotions. American Psychologist, 56, 218-226. doi:10.1037/0003-066X.56.3.218.

Gollwitzer, P. M. (1999). Implementation intentions: Strong effects of simple plans. American Psychologist, 54(7), 493-503. doi:10.1037/0003-066X.54.7.493.

Gross, J. J. (1998). The emerging field of emotion regulation: An integrative review. Review of General Psychology, 2, 271-299. doi:10.1037/1089-2680.2.3.271.

Hanssen, M. M., Vancleef, L. M. G., Vlaeyen, J. W. S., Hayes, A. F., Schouten, E. G. W., \& Peters, M. L. (2016). Optimism, motivational coping and well-being: Evidence supporting the importance of flexible goal adjustment. Journal of Happiness Studies. doi:10.1007/s10902-014-9572-x.

Heckhausen, H., \& Gollwitzer, P. M. (1987). Thought contents and cognitive functioning in motivational versus volitional states of mind. Motivation and Emotion, 11, 101-120. doi:10.1007/BF00992338.

Ilies, R., De Pater, I. E., \& Judge, T. (2007). Differential affective reactions to negative and positive feedback, and the role of self-esteem. Journal of Managerial Psychology, 22, 590-609. doi:10.1108/ 02683940710778459. 
Judge, T. A., Ilies, R., \& Scott, B. A. (2006). Work-family conflict and emotions: Effects at work and at home. Personnel Psychology, 59(4), 779-814. doi:10.1111/j.1744-6570.2006.00054.x.

Kline, R. B. (2012). Principles and practice of structural equation modeling. New York: Guilford Press.

Koestner, R., Otis, N., Powers, T. A., Pelletier, L., \& Gagnon, H. (2008). Autonomous motivation, controlled motivation, and goal progress. Journal of Personality, 76(5), 1201-1230. doi:10.1111/j.14676494.2008.00519.x.

Laguna, M., Alessandri, G., \& Caprara, G. V. (2016). Personal goal realization in entrepreneurs: A multilevel analysis of the role of affect and positive orientation. Applied Psychology: An International Review. doi:10.1111/apps.12061.

Lamiell, J. T. (1981). Toward an idiothetic psychology of personality. American Psychologist, 36, $276-289$. doi:10.1037/0003-066X.36.3.276.

Little, B. (1999). Personality and motivation: Personal action and the conative revolution. In L. A. Pervin \& O. P. John (Eds.), Handbook of personality: Theory and research (2nd ed., pp. 501-524). New York: The Guilford Press.

Little, B. R. (2006). Personality science and self-regulation: Personal projects as integrative units. Applied Psychology: An International Review, 55, 419-427. doi:10.1111/j.1464-0597.2006.00262.x.

Little, B. R., \& Gee, T. L. (2007). The methodology of personal projects analysis: four modules and a funnel. In B. R. Little, K. Salmela-Aro, \& S. D. Phillips (Eds.), Personal project pursuit: goals, action, and human flourishing (pp. 51-93). New York: Lawrence Erlbaum.

Ludtke, O., Marsh, H. W., Robitzsch, A., Trautwein, U., Asparouhov, T., \& Muthén, B. (2008). The multilevel latent covariate model: A new, more reliable approach to group-level effects in contextual studies. Psychological Methods, 13, 203-229. doi:10.1037/a0012869.

Lüdtke, O., \& Trautwein, U. (2007). Aggregating to the between-person level in idiographic research designs: Personal goal research as an example of the need to distinguish between reliability and homogeneity. Journal of Research in Personality, 41, 230-238. doi:10.1016/j.jrp.2006.03.005.

Lyubomirsky, S., King, L., \& Diener, E. (2005). The benefits of frequent positive affect: Does happiness lead to success? Psychological Bulletin, 131, 803-855. doi:10.1037/0033-2909.131.6.803.

McGregor, I., McAdams, D. O., \& Little, B. R. (2006). Personal projects, life stories, and happiness: On being true to traits. Journal of Research in Personality, 40, 551-572. doi:10.1016/j.jrp.2005.05.002.

Milyavskaya, M., Nadolny, D., \& Koestner, R. (2014). Where do self-concordant goals come from? The role of domain-specific psychological need satisfaction. Personality and Social Psychology Bulletin, 40(6), 700-711. doi:10.1177/0146167214524445.

Monzani, D., Steca, P., Greco, A., D’Addario, M., Pancani, L., \& Cappelletti, E. (2015). Effective pursuit of personal goals: The fostering effect of dispositional optimism on goal commitment and goal progress. Personality and Individual Differences, 82, 203-214. doi:10.1016/j.paid.2015.03.019.

Muthen, B., \& Satorra, A. (1995). Complex sample data in structural equation modeling. Sociological Methodology, 25, 267-316.

Nezlek, J. B. (2007). A multilevel framework for understanding relationships among traits, states, situations, and behaviors. European Journal of Personality, 21, 789-810. doi:10.1002/per.640.

Nezlek, J. B. (2012). Multilevel modeling for psychologists. In H. Cooper, P. M. Camic, D. L. Long, A. T. Panter, D. Rindskopf, \& K. J. Sher (Eds.), APA handbook of research methods in psychology (Vol. 3, pp. 219-241). Washington, DC: American Psychological Association.

Nurmi, J.-E., Salmela-Aro, K., \& Aunola, K. (2009). Personal goal appraisals vary across both individuals and goal contents. Personality and Individual Differences, 47, 498-503. doi:10.1016/j.paid.2009.04. 028.

Oleś, P. K., Alessandri, G., Oleś, M., Bak, W., Jankowski, T., Laguna, M., \& Caprara, G. V. (2013). Positive orientation and generalized self-efficacy. Studia Psychologica, 1, 47-59.

Plemmons, S. A., \& Weiss, H. M. (2013). Goals and affect. In E. A. Locke \& G. P. Latham (Eds.), New developments in goal setting and task performance (pp. 117-132). New York: Routledge.

Pomaki, G., Maes, S., \& terDoest, L. (2004). Work conditions and employees' self-set goals: Goal processes enhance prediction of psychological distress and well-being. Personality and Social Psychology Bulletin, 30, 685-694.

Posner, J., Russell, J. A., \& Peterson, B. S. (2005). The circumplex model of affect: An integrative approach to affective neuroscience, cognitive development, and psychopathology. Development and Psychopathology, 17(3), 715-734. doi:10.1017/S0954579405050340.

Rosenberg, M. (1965). Society and the adolescent self-image. Princeton: Princeton Univ. Press.

Russell, J. A. (1980). A circumplex model of affect. Journal of Personality and Social Psychology, 39(6), 1161-1178. doi:10.1037/h0077714. 
Salanova, M., Llorens, S., \& Schaufeli, W. B. (2011). "Yes, I can, I feel good, and I just do it!” On gain cycles and spirals of efficacy beliefs, affect, and engagement. Applied Psychology: An International Review, 60, 255-285. doi:10.1111/j.1464-0597.2010.00435.x.

Salmela-Aro, K. (2009). Personal goals and well-being during critical life transitions: The four C'schannelling, choice, co-agency and compensation. Advances in Life Course Research, 14(1-2), 63-73. doi:10.1016/j.alcr.2009.03.003.

Salmela-Aro, K., Aunola, K., \& Nurmi, J.-E. (2007). Personal goals during emerging adulthood: A 10-year follow up. Journal of Adolescent Research, 22(6), 690-715. doi:10.1177/0743558407303978.

Salmela-Aro, K., Näätänen, P., Tolvanen, A., \& Nurmi, J. E. (2011). Changes in goal-related affects: Decrease burnout during a group psychotherapy intervention. The European Journal of Work and Organizational Psychology, 20, 451-466. doi:10.1080/13594321003633703.

Scheier, M. F., Carver, C. S., \& Bridges, M. W. (1994). Distinguishing optimism from neuroticism (and trait anxiety, self-mastery, and self-esteem): A reevaluation of the Life Orientation Test. Journal of Personality and Social Psychology, 67, 1063-1078. doi:10.1037/0022-3514.67.6.1063.

Seo, M.-G., Bartunek, J. M., \& Barrett, L. F. (2010). The role of affective experience in work motivation: Test of a conceptual model. Journal of Organizational Behavior, 31(7), 951-968. doi:10.1002/job.655.

Sheldon, K. M., \& Elliot, A. J. (2000). Personal goals in social roles: Divergences and convergences across roles and levels of analysis. Journal of Personality, 68, 51-84. doi:10.1111/1467-6494.00091.

Sheldon, K. M., Kasser, T., Smith, K., \& Share, T. (2002). Personal goals and psychological growth: Testing an intervention to enhance goal-attainment and personality integration. Journal of Personality, 70, 5-31. doi:10.1111/1467-6494.00176.

Sitzmann, T., \& Yeo, G. (2013). A meta-analytic investigation of the within-person self-efficacy domain: Is self-efficacy a product of past performance or a driver of future performance? Personnel Psychology, 66(3), 531-568. doi:10.1111/peps.12035.

Sobol-Kwapinska, M., \& Jankowski, T. (2015). Positive time: Balanced time perspective and positive orientation. Journal of Happiness Studies. doi:10.1007/s10902-015-9656-2.

Steca, P., Monzani, D., Greco, A., D’Addario, M., Cappelletti, E., \& Pancani, L. (2015). The effects of short-term personal goals on subjective well-being. Journal of Happiness Studies. doi:10.1007/s10902015-9651-7.

Wiese, B. S., \& Freund, A. M. (2005). Goal progress makes one happy, or does it? Longitudinal findings from the work domain. Journal of Occupational and Organizational Psychology, 78, 287-304. doi:10. 1348/096317905X26714.

Wrosch, C., Scheier, M. F., Carver, C. S., \& Schulz, R. (2003). The importance of goal disengagement in adaptive self-regulation: When giving up is beneficial. Self and Identity, 2, 1-20. doi:10.1080/ 15298860309021.

Zięba, M. (2012). Samoocena i nadzieja a formalne właściwości projektów osobistych [Self-esteem, hope and formal characteristics of personal projects]. Przeglad Psychologiczny, 55, 161-178. 